\title{
SUBLETHAL PESTICIDE CONCENTRATIONS AND PREDATION JOINTLY SHAPE LIFE HISTORY: BEHAVIORAL AND PHYSIOLOGICAL MECHANISMS
}

\author{
Melina Campero, ${ }^{1,2,3}$ Stefanie Slos, ${ }^{1}$ Frans Ollevier, ${ }^{1}$ and Robby Stoks ${ }^{1}$ \\ ${ }^{1}$ Katholieke Universiteit Leuven (KULeuven), Laboratory of Aquatic Ecology, Ch. Deberiotstraat 32, B-3000, Leuven, Belgium \\ ${ }^{2}$ Universidad Mayor de San Simon (UMSS), Unidad de Limnologia y Recursos Acuaticos (ULRA), Parque La Torre, \\ calle Sucre s/n. Casilla 1486, Cochabamba, Bolivia
}

\begin{abstract}
Despite their relevance for risk assessment, the interactive effects of pesticide and predation cues are poorly understood because the underlying behavioral and physiological mechanisms are largely unknown. To explore these mechanisms, we reared larvae of the damselfly Coenagrion puella at three different predation risk levels and a range of environmentally realistic concentrations of three pesticides used worldwide (atrazine, carbaryl, and endosulfan). We compared key development responses (growth rate, developmental time, and final size) against food ingestion, assimilation, and conversion efficiency, and acetylcholinesterase (AChE) activity. Predation risk impaired all endpoints, including AChE activity, while the effects of pesticide stress were smaller for atrazine and endosulfan and absent for carbaryl. The effects of both stressors and their interaction on life history were mostly indirect through resource acquisition and energy allocation. Compensatory physiological mechanisms to pesticide stress (atrazine and endosulfan) were present in larvae reared in the absence of predation stress but were offset under predation stress. As a result, smaller size (atrazine and endosulfan) and lower growth rate (endosulfan) from pesticide stress were only found in the highest predation risk treatment. Our results provide insight as to the conditions under which interactions between stressors are likely to occur: damselfly populations at high density and living in fish ponds will be more affected by pesticides than populations at low densities in fishless ponds. By identifying variables that may shape the interaction between predation stress and other stressors such as pesticides, our mechanistic approach may help to bridge the gap between laboratory and field studies.
\end{abstract}

Key words: acetylcholinesterase activity; Coenagrion puella; compensatory mechanisms; damselfy; interactions between stressors; pesticide stress; predator stress; structural equation models.

\section{INTRODUCTION}

The focus in stress ecology, with stress being an environmental condition that impairs fitness (Sibly and Calow 1989), shifted from studies where effects of environmental stressors were tested individually, toward studies where effects of combined stressors are also evaluated. This is because organisms are often simultaneously exposed to several stressors, and combined stressors often do not generate additive effects (Sih et al. 2004). Ignoring synergism among stressors may have significant implications by underestimating their negative effects. A synergism that recently received wide attention is the one between pesticide stress and stress imposed by predators (Relyea and Mills 2001, Relyea 2003). This has potentially large implications for environmental management and the worldwide decline of species (Storfer 2003).

There is an increasing number of studies reporting the synergistic effects of pesticide and predation stress on

Manuscript received 14 March 2007; accepted 17 April 2007. Corresponding Editor: R. A. Relyea.

${ }^{3}$ E-mail: melina.camperopaz@bio.kuleuven.be mortality and life history that identified predatory consumption and direct intraspecific and interspecific interactions as the underlying mechanisms (e.g., Dewey 1986, Mills and Semlitsch 2004, Chang et al. 2005, Rohr and Crumrine 2005). Studies have also shown that only predator cues (without predatory consumption and other biotic interactions) may generate synergistic mortality and life history effects (Relyea and Mills 2001, Relyea 2003, 2004, 2005). The mechanisms underlying these synergistic effects are less understood. Relyea and Mills (2001) showed parallel changes in activity that suggest a behavioral component may play a role. Food ingestion is a key behavioral variable linked to life history, but synergism studies directly measuring this variable are lacking. Further, to the best of our knowledge, there are no studies focusing on physiological mechanisms potentially associated with the interaction between pesticide and predation stress. Studies focusing on behavioral and physiological mechanisms can help us to better understand the mechanistic basis of the interaction between pesticide and predation stress and eventually to predict its occurrence (Sih et al. 2004, Teplitsky et al. 2005). 
Candidate behavioral and physiological mechanisms that may underlie the synergism are those already known to be affected by both stressors separately. Pesticides can have negative effects on life history by one of three mechanisms: (1) reducing food intake (Ribeiro et al. 2001); (2) diverting energy to organism maintenance (Congdon et al. 2001); and (3) modifying the action and levels of target biomolecules in the organism (e.g., inhibition of enzymatic activity, reduction of energy storage level [Newman 1998]). These three mechanisms have also been identified to underlie the negative effects of predation stress on life history. There is substantial evidence that predators negatively affect life history of their prey organisms by reducing food intake (reviewed by Lima 1998), and recent studies suggest this also works through a reduced allocation of acquired resources to growth (McPeek et al. 2001, McPeek 2004, Stoks et al. 2005b). Finally, it is known that predation stress may affect biomolecules, for example induce costly heat shock proteins (Pauwels et al. 2005), reduce energy storage levels (Stoks et al. $2005 a$ ) or increase cortisol levels (Boonstra et al. 1998).

In this study, we aim to identify potential synergisms between pesticide stress and predation stress for several life history traits (i.e., development time, growth rate, and final size) and to explore the behavioral and physiological mechanisms that may underlie the occurrence of the interaction between both stressors. We studied endpoints directly linked with each of the three previously mentioned mechanisms (i.e., food intake, energy allocation, and modification/production of biomolecules). We selected acetylcholinesterase (AChE) activity as the target biomolecule in this study, as it is inhibited by several pesticides (Adams 2001) and may affect behavior and even survival (Fleischli et al. 2004). Barry (2002) reported that cholinesterase inhibitors synergistically interacted with predator cues to induce maximal expression of a morphological antipredator trait in Daphnia pulex. Further, it has been shown that AChE activity is responsible for behavior modification observed in individuals exposed to pesticide stress (Rao et al. 2005, Sandahl et al. 2005).

We studied these response variables in larvae of the damselfly Coenagrion puella exposed to different levels of pesticide stress at different predation risk levels. We predicted that predation risk, pesticide stress, and their interaction would negatively affect life history through one or more of the following mechanisms: reduced food intake, changes in energy allocation, or inhibition of AChE. To evaluate the generality of our findings, we used three common pesticides: atrazine (herbicide), and carbaryl and endosulfan (insecticides).

\section{Methods}

\section{Collection of adults and larvae rearing}

Eighteen mating pairs of the damselfly Coenagrion puella (Linnaeus) were collected in June 2004 at a small pond in Oud-Heverlee (Belgium). Each pair was placed in a separate 3 -L glass jar containing wet filter paper as an oviposition substrate. Freshly hatched larvae were randomly allocated in groups of 30 to plastic containers $(20 \times 15 \times 10 \mathrm{~cm})$ containing moderately soft reconstituted water made following the protocol of Weber (1993). Containers were placed in a room at $23^{\circ} \mathrm{C}$ (range $20-25^{\circ} \mathrm{C}$ ) with a constant photoperiod of $16 \mathrm{~L}: 8 \mathrm{D}$. Larvae were fed twice a day ad libitum with Artemia salina nauplii for the entire duration of the experiment.

\section{Pesticide solutions}

Three pesticides, atrazine, carbaryl, and endosulfan, were chosen on the basis of their use in Belgium and were bought from Sigma Aldrich (Pestanal class, purity $>$ 98.9\%; Sigma Aldrich, Bornem, Belgium). Pesticides were dissolved in acetone and diluted in reconstituted water ( $\mathrm{pH} 7.2)$ to prepare stock solutions of $100000 \mathrm{ppb}$ $(1 \mathrm{mg} / 10 \mathrm{~mL}$ acetone; $1 \mathrm{ppb}=1 \mu \mathrm{g} / \mathrm{L})$, kept in a dark room at $4^{\circ} \mathrm{C}$. Atrazine is an herbicide used to treat corn crops in Belgium. In surface waters around corn fields, concentrations up to $400 \mathrm{ppb}$ have been found (Steurbaut et al. 2003). Based on those data and on a pilot experiment, we opted for the following three concentrations to ensure nonlethal effects: 5, 40, and $320 \mathrm{ppb}$. Carbaryl is an insecticide from the carbamate group, which in high doses is well known as an inhibitor of the enzyme acetylcholinesterase (AChE; Thompson 1999). Previous studies on damselflies showed increased asymmetry at $10 \mathrm{ppb}$ and no emergence at $100 \mathrm{ppb}$ (Hardersen and Wratten 1998). As our aim was to work with sublethal concentrations and a pilot experiment showed high mortality of two-week-old larvae at $20 \mathrm{ppb}$, the chosen experimental concentrations were 1, 5, and $10 \mathrm{ppb}$. Endosulfan is an organochlorine insecticide for which concentrations higher than $100 \mathrm{ppb}$ have been found in surface waters in Belgium (Steurbaut et al. 2003). Previous studies showed higher mortality in Ambystoma barbouri salamanders and macroinvertebrate drift for concentrations lower than $8.2 \mathrm{ppb}$ (Hose et al. 2003, Rohr et al. 2003). Initially, the chosen concentrations were 1,5 , and $10 \mathrm{ppb}$, but all the larvae died in the highest two concentrations in a pilot experiment of $96 \mathrm{~h}$ conducted on two-week-old larvae. Therefore, we opted for the following concentrations: $0.1,1$, and $3 \mathrm{ppb}$. Note that our aim was not to directly compare pesticides, so the use of different concentrations was not important.

The concentrations used for the three pesticides were below the $\mathrm{LC}_{50}$ for damselflies according to the Pesticide Action Network Pesticide Database (available online). ${ }^{4}$ In line with this, no differences in mortality between controls and treatments were found. Pesticide solutions were renewed every seven days in all cups. This was also done for controls to correct for any effects of disturbance. Analysis of the pesticide samples by means

\footnotetext{
${ }^{4}\langle$ http://www.pesticideinfo.org $\rangle$
} 
of high-pressure liquid chromatography (HPLC) revealed that the actual starting concentrations were 5.7, 37.6, and $323.1 \mathrm{ppb}$ for atrazine, 1.5, 5.3, and $9.8 \mathrm{ppb}$ for carbaryl, and $0.9,1.7$, and 5.2 ppb for endosulfan. The degradation rate for atrazine and endosulfan after seven days was $\sim 4.5 \%$ and $70 \%$, respectively. For carbaryl, the degradation rate was $100 \%$ for the lowest concentration and up to $90 \%$ for the medium and highest concentrations after seven days. Oxygen concentrations (mean = $7.06 \mathrm{mg} / \mathrm{L} ; 74 \%$ saturation), temperature (mean = $\left.23.8^{\circ} \mathrm{C}\right)$, and $\mathrm{pH}($ mean $=7.39)$ were not influenced by treatments (all $P>0.10)$.

\section{Experimental design}

To test for effects of predation risk on the sublethal effects of pesticides, we used a completely randomized 3 $\times 3 \times 3$ design with three concentrations for each of the three pesticides crossed with three predation risk levels. Thirty individuals were assigned to each pesticide $\times$ concentration $\times$ predation risk combination, which gives a total of 270 larvae per pesticide (30 larvae $\times 3$ concentrations $\times 3$ predation risk levels). Additionally, 30 larvae were reared as solvent control and 30 larvae as water control per predation risk level. This gives a total of 180 control larvae $(30$ larvae $\times 2$ controls $\times 3$ predation risk levels). The experiment ran for 104 days.

At the start of the experiment, two weeks after hatching, all larvae were individually placed in cylindrical $60-\mathrm{mL}$ plastic vials $(7 \mathrm{~cm}$ height, $3.5 \mathrm{~cm}$ diameter, water height $3 \mathrm{~cm}$ ). Pesticide concentrations were randomly assigned to each vial. Groups of four randomly selected vials were placed within $2-\mathrm{L}$ aquaria $(20 \times 15 \times 17 \mathrm{~cm})$. This gives a density of 133 individuals $/ \mathrm{m}^{2}$, which is well within the natural observed range for coenagrionid damselflies (Banks and Thompson 1987, Gribbin and Thompson 1990). Vials were fixed at $3 \mathrm{~cm}$ above the bottom of the aquaria in a metal frame, and the aperture of the vials was $2 \mathrm{~cm}$ above the water level of the aquaria to avoid water exchange between the vials and the aquaria. Different sets of vials were made on a weekly basis during the water renewal, by randomly reshuffling vials across aquaria (i.e., there were no fixed sets of vials, and each individual vial was not matched to a certain aquarium) to avoid pseudoreplication (see McPeek 2004).

We installed three levels of predation risk: larvae were reared in isolation, together with conspecifics, and together with conspecifics and a fish predator. The isolation treatment reflects a situation without predation risk. To achieve this, the vials were made opaque with tape to prevent any visual interaction with adjacent larvae. In the conspecifics treatment the vials were left transparent. This assured visual contact with conspecific larvae but avoided any physical contact. As damselflies are cannibalistic, they impose predator stress when they can see each other (McPeek et al. 2001, Stoks and McPeek 2003). As fish are important predators of damselfly larvae (McPeek 1990), we also included a fish treatment in which one pumpkinseed Lepomis gibossus (average size from head to tail: $4 \mathrm{~cm}$ ) was placed within the 2-L aquaria. This way, larvae could see each other and the fish predator without the possibility of cannibalism or direct predation. A pilot experiment using growth rate as response variable, showed that similar stress levels were achieved when the predator was perceived by the damselflies only by sight (i.e., visual cues) or by a sight $\times$ predator cues combination (i.e., adding $1 \mathrm{~mL}$ of water with fish cues into each individual vial; M. Campero, unpublished data). Therefore, we decided to use only visual perception of the predator as stressor.

\section{Response variables}

We quantified three life history traits. As the experiment was stopped before emergence, development time was calculated as the number of days from hatching until a larva entered the $\mathrm{F}-2$ stage, with $\mathrm{F} 0$ being the final instar. Growth rate was calculated as $(\ln$ [final wet mass] - $\ln$ [initial wet mass]/104 days), where initial mass was estimated as the average wet mass of 30 larvae at the beginning of the experiment. Final wet mass was taken at the end of the experiment. Each larva was gently blotted dry and weighed to the nearest $0.01 \mathrm{mg}$ using an electronic microbalance (Mettler Toledo, Sint-StevensWoluwe, Belgium). Head width was calculated from images taken using a stereomicroscope connected to the imaging software Image Pro Plus version 5.0 (Photometrics UK, Marlow, UK). Head width is the standard approximation for total size in damselflies (Corbet 1999).

At the end of the experiment each larva was individually stored in a $-80^{\circ} \mathrm{C}$ freezer until $\mathrm{AChE}$ analysis. Enzyme activity was measured spectrophotometrically on individual head homogenates using a modification of the Ellman method (Jensen et al. 1997) standardized for C. puella (S. Slos, unpublished data).

Food intake, assimilation, and conversion efficiency were determined based on a mass budget (following McPeek et al. 2001) for six days starting one day after the larva entered the F-2 stage. Every day, a sample of the food, the uneaten food, and the fecal pellets per larva were kept for subsequent analyses. These samples were dried at $60^{\circ} \mathrm{C}$ for $48 \mathrm{~h}$ and weighed on a Cahn electrobalance (Cahn, Breda, The Netherlands) to the nearest $0.1 \mu \mathrm{g}$. The amount of ingested food by a single larva was obtained by the difference of the total mass of given food to that larva and the total mass of uneaten food of that larva. The amount of assimilated food was calculated as the difference in total mass of ingested food and total mass of fecal pellets. Assimilation efficiency was taken as the amount of assimilated food divided by the amount of ingested food. Conversion efficiency was obtained as $(\ln$ [final wet mass] - $\ln$ [initial wet mass])/amount of assimilated food during the sixday period (McPeek et al. 2001). To calculate conversion efficiency, no differences are found when using larval 


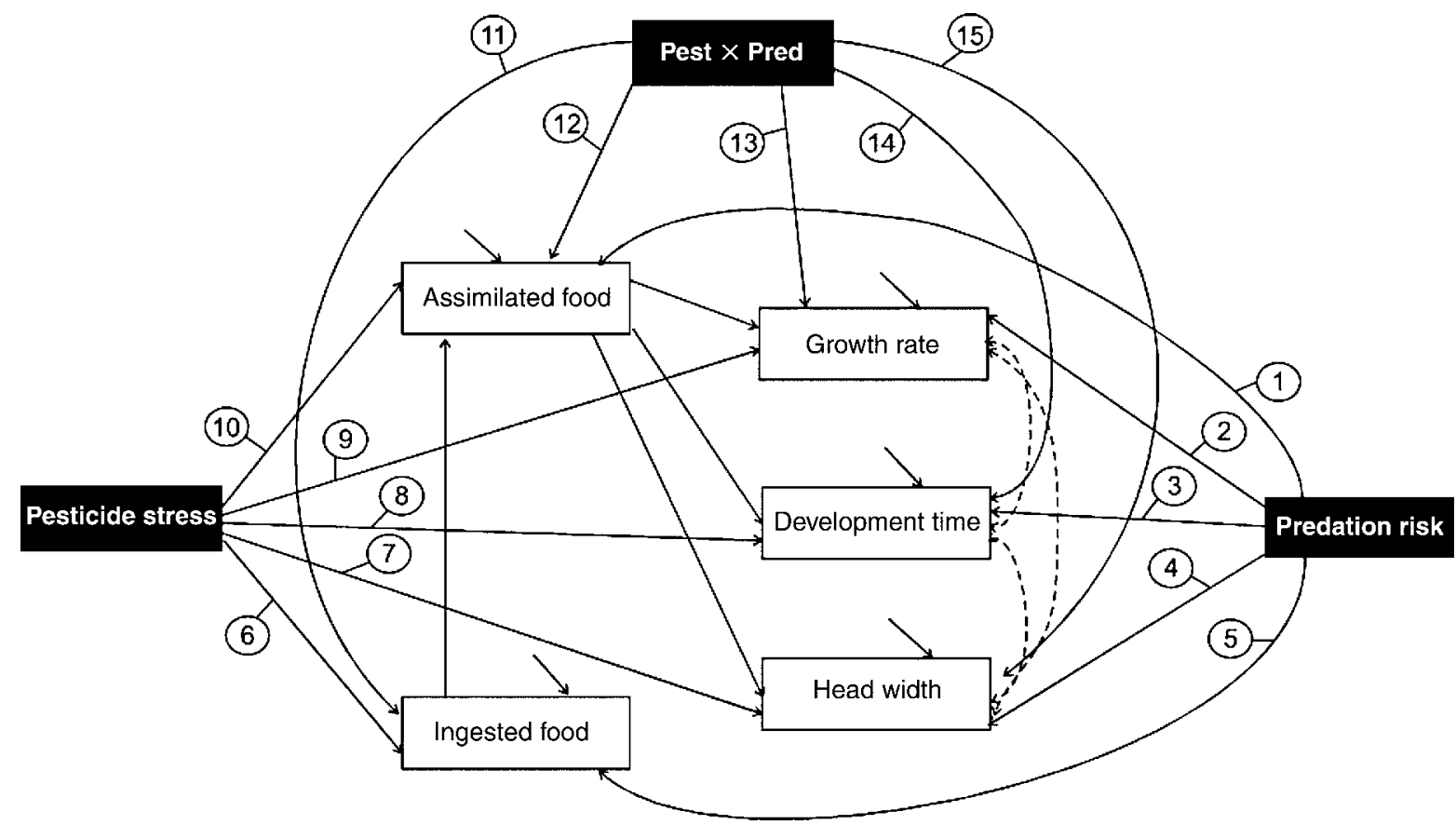

FIG. 1. Hypothesized structural equation model (SEM) for direct effects of predation risk and pesticide stress (Pest $\times$ Pred) on life history traits. Paths 1-15 depict the direct effects of the stressors on the response variables. Solid one-headed arrows represent causal effects of one variable on another variable. Dashed double-headed arrows represent correlation between variables. Arrows not originating from a variable represent residual error variances (not shown in Fig. 4).

wet mass or dry mass, as in coenagrionid damselflies dry mass is directly proportional to wet mass (McPeek et al. 2001).

\section{Statistical analyses}

No differences between water and solvent control for any of the response variables were found $(P>0.1)$. Therefore the data for the water and solvent control were pooled into a single treatment (concentration " 0 "). To look for main effects and an interaction between pesticide stress and predation stress, we ran multivariate analyses of covariance (MANCOVA) for each pesticide with predation risk as the categorical predictor variable, pesticide concentration as the continuous predictor variable, and all seven response variables (i.e., growth rate, development time, size, ingested food, assimilation efficiency, conversion efficiency, and AChE activity) as the dependent variables. The interaction between predation risk and pesticide stress was also included in the model. To interpret the MANCOVA, separate ANCOVAs for each response variable were run. Pesticide concentrations were log-transformed to fulfill the linearity assumption of the analyses. When the effects of predation risk were significant, we carried out corrected Tukey-Kramer post hoc tests to identify which levels differed from each other. All analyses were run in SAS version 9.12.

The ANCOVAs tested for effects of predation risk and pesticide concentrations on each endpoint separately without taking the hierarchical links among enzymatic activity, digestive physiology, and life history into account. As our aim was also to identify mechanistic pathways that may explain the occurrence of a synergism between both stressors, we used structural equation models (SEM) to quantify the strength and the sign of the effect of both stressors on life history. The effects of a variable on another one are quantified as path coefficients, which basically are partial multiple regression coefficients. Path coefficients were standardized because standardization converts all paths to a common metric that is useful to compare their relative importance (Pugesek 2003).

Our SEM model accounts for both direct and indirect effects of predation risk, pesticide stress, and their interaction on the measured life history traits. Direct effects account for the effects of the stressors on the life history endpoints without involving food ingestion or food assimilation (paths 1-15, Fig. 1). Indirect effects refer to the effects of the stressors on life history channeled through food ingestion and food assimilation. Strength and sign of the total effect of a stressor on a life history trait can be calculated by summing both the direct and indirect effects (for further explanation of direct, indirect, and total effects, see Appendix). Goodness-of-fit measures of early models suggested that no link between AChE activity and digestive physiology/life history existed. Therefore, AChE activity was left out of the SEM models. Growth rate for the six-day period during which all digestive variables were calculated and the growth rate of the 104 days of the 
TABLE 1. Results of ANCOVAs testing for effects of predation stress and pesticide concentrations on life history variables, food ingestion, assimilation efficiency, conversion efficiency and AChE activity.

\begin{tabular}{|c|c|c|c|c|c|c|c|c|c|}
\hline \multirow[b]{2}{*}{ Endpoint } & \multicolumn{3}{|c|}{ Atrazine } & \multicolumn{3}{|c|}{ Carbaryl } & \multicolumn{3}{|c|}{ Endosulfan } \\
\hline & df & $F$ & $P$ & $\mathrm{df}$ & $F$ & $P$ & df & $F$ & $P$ \\
\hline \multicolumn{10}{|l|}{$\begin{array}{l}\text { Life history } \\
\text { Growth rate }\end{array}$} \\
\hline Pesticide stress & 1,109 & 2.11 & 0.15 & 1,128 & 0.26 & 0.61 & 1,175 & 4.65 & 0.03 \\
\hline Predation risk & 2,109 & 224.24 & $<0.0001$ & 2,128 & 298.33 & $<0.0001$ & 2,175 & 580.92 & $<0.0001$ \\
\hline Pest $\times$ pred & 2,109 & 0.21 & 0.81 & 2,128 & 0.30 & 0.73 & 2,175 & 15.78 & $<0.0001$ \\
\hline \multicolumn{10}{|l|}{ Development time } \\
\hline Pesticide stress & 1,109 & 0.01 & 0.94 & 1,128 & 0.00 & 0.99 & 1,175 & 1.96 & 0.16 \\
\hline Predation risk & 2,109 & 45.35 & $<0.0001$ & 2,128 & 41.69 & $<0.0001$ & 2,175 & 60.53 & $<0.0001$ \\
\hline Pest $\times$ pred & 2,109 & 2.20 & 0.11 & 2,128 & 0.58 & 0.56 & 2,175 & 0.40 & 0.67 \\
\hline \multicolumn{10}{|l|}{ Head width } \\
\hline Pesticide stress & 1,109 & 3.53 & 0.06 & 1,128 & 1.33 & 0.25 & 1,175 & 2.79 & 0.09 \\
\hline Predation risk & 2,109 & 1.79 & 0.17 & 2,128 & 3.21 & 0.043 & 2,175 & 3.51 & 0.03 \\
\hline Pest $\times$ Pred & 2,109 & 0.71 & 0.49 & 2,128 & 2.51 & 0.08 & 2,175 & 3.48 & 0.03 \\
\hline \multicolumn{10}{|l|}{ Behavior } \\
\hline \multicolumn{10}{|l|}{ Food ingestion } \\
\hline Pesticide stress & 1,109 & 0.93 & 0.33 & 1,128 & 0.13 & 0.71 & 1,175 & 2.01 & 0.15 \\
\hline Predation risk & 2,109 & 224.28 & $<0.0001$ & 2,128 & 255.75 & $<0.0001$ & 2,175 & 403.73 & $<0.0001$ \\
\hline Pest $\times$ pred & 2,109 & 1.16 & 0.31 & 2,128 & 0.59 & 0.55 & 2,175 & 10.17 & $<0.0001$ \\
\hline \multicolumn{10}{|c|}{ Physiology } \\
\hline \multicolumn{10}{|c|}{ Assimilation efficiency } \\
\hline Pesticide stress & 1,109 & 8.50 & 0.004 & 1,128 & 3.83 & 0.05 & 1,175 & 5.5 & 0.02 \\
\hline Predation risk & 2,109 & 329.14 & $<0.0001$ & 2,128 & 377.17 & $<0.0001$ & 2,175 & 614.75 & $<0.0001$ \\
\hline Pest $\times$ pred & 2,109 & 4.81 & 0.03 & 2,128 & 0.00 & 0.99 & 2,175 & 9.4 & 0.0001 \\
\hline \multicolumn{10}{|c|}{ Conversion efficiency } \\
\hline Pesticide stress & 1,109 & 6.2 & 0.014 & 1,128 & 2.91 & 0.09 & 1,175 & 4.19 & 0.04 \\
\hline Predation risk & 2,109 & 6.77 & 0.01 & 2,128 & 6. 90 & 0.01 & 2,175 & 5.51 & 0.02 \\
\hline Pest $\times$ pred & 2,109 & 0.69 & 0.5 & 2,128 & 0.35 & 0.55 & 2,175 & 3.89 & 0.05 \\
\hline \multicolumn{10}{|l|}{ AChE activity } \\
\hline Pesticide stress & 1,109 & 0.87 & 0.35 & 1,128 & 0.02 & 0.90 & 1,175 & 2.48 & 0.12 \\
\hline Predation risk & 2,109 & 7.20 & 0.001 & 2,128 & 6.63 & 0.002 & 2,175 & 10.76 & $<0.0001$ \\
\hline Pest $\times$ pred & 2,109 & 5.33 & 0.006 & 2,128 & 0.16 & 0.85 & 2,175 & 0.41 & 0.66 \\
\hline
\end{tabular}

Note: The interaction between predation risk and pesticide is represented as "Pest $\times$ pred."

experiment were highly correlated $(r=0.92, P<0.0001)$. Therefore, we only report in the Results growth rates based on the six-day period.

Predation risk was used as an ordinal variable, with values of 1 (isolated larvae), 2 (larvae reared with conspecifics), and 3 (larvae reared with conspecifics and a fish predator). Pesticide exposure is a continuous variable, where the pesticide concentrations were used as values. The interaction between predation risk and pesticide stress was modeled following Yang (1998). Covariation between life history variables, which often takes the form of trade-offs, was implemented in the models. To formally test the fit of the path models to our data, we used three goodness-of-fit measures: the $\chi^{2}$ value that tests for the similarity between the observed (data based) and the predicted covariance matrix (model based); the root mean square error of approximation (RMSEA) where values $\leq 0.05$ indicate a good fit, and the adjusted goodness-of-fit index (AGFI) where values $\geq 0.90$ reflect good fit. All structural equation model analyses were performed with LISREL version 8.72 (Jöreskog and Sörbom 2004).

\section{RESUlts}

\section{Predation stress}

The MANCOVA showed that predation stress had an overall negative influence on the set of response variables (atrazine, Wilks' $\lambda=0.075, F_{12,208}=46, P<$ 0.001 ; carbaryl, Wilks' $\lambda=0.067, F_{12,246}=58.83, P<$ 0.001 ; endosulfan, Wilks' $\lambda=0.051, F_{12,340}=97.35, P<$ 0.001). The separate ANCOVAs showed that all measured endpoints (with the exception of head width in the atrazine treatment) were affected by predation risk (Table 1). Isolated larvae show higher values for all endpoints and shorter development time than larvae from the conspecifics and fish predator treatments (Tukey-Kramer tests: all $P<0.0001$; Figs. 2 and 3). The latter two predation risk levels did not differ for any endpoint (Tukey-Kramer tests: all $P>0.10$ ), except for head width in the endosulfan treatment $\left(t_{175}=2.24, P=\right.$ 0.043 ). Especially noteworthy is the up to $70 \%$ decrease in AChE activity in the conspecifics and fish treatment compared to the isolation treatment (Fig. 3). 
○ Isolation

Atrazine
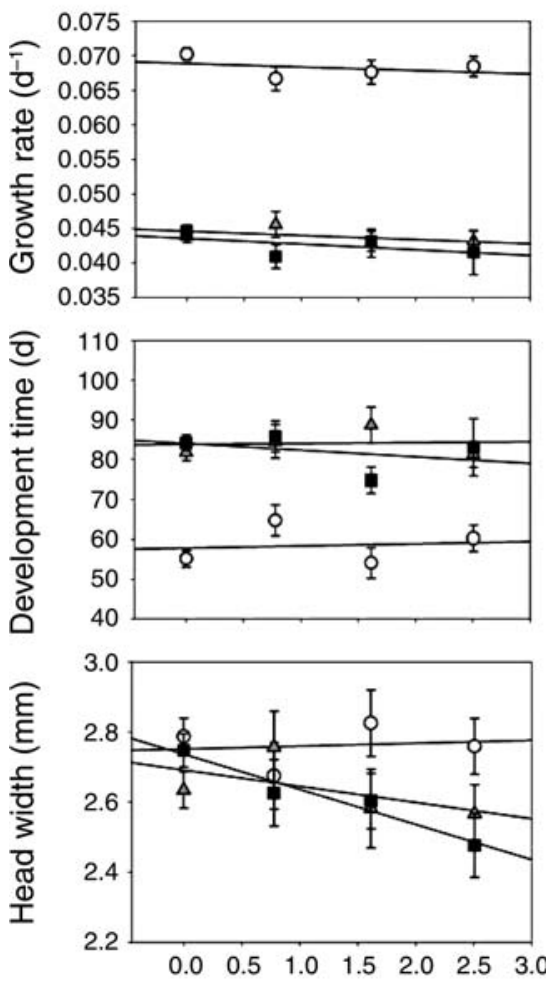

$\Delta$ Conspecifics

Carbaryl
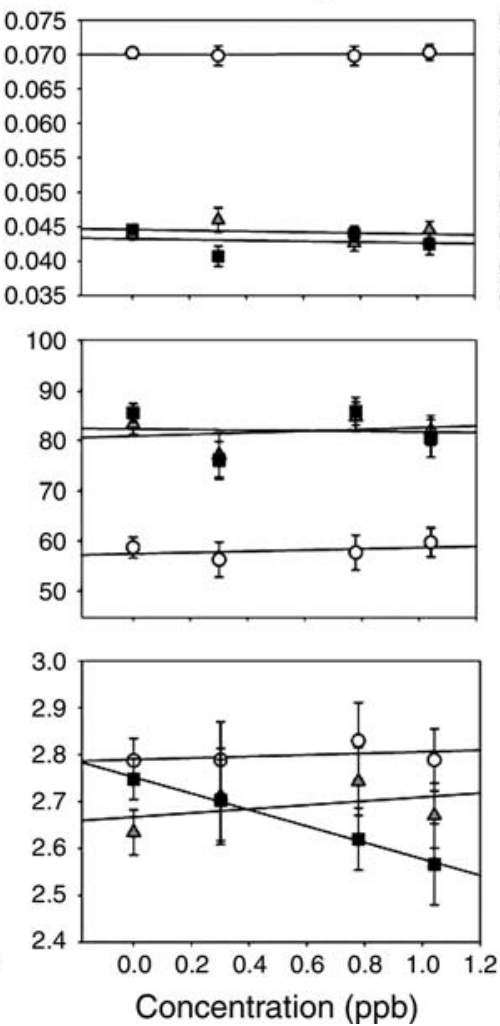

- Conspecifics + fish

Endosulfan
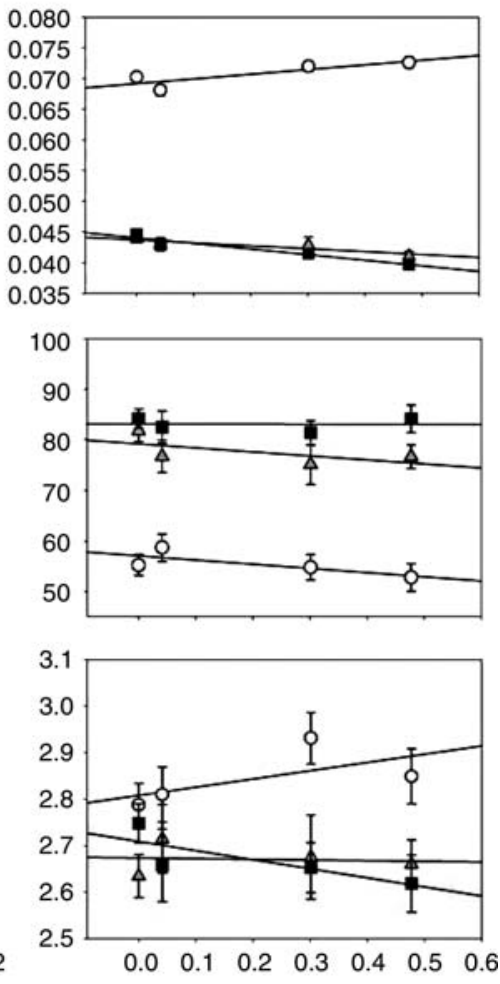

FIG. 2. Effects of pesticide concentrations and predation stress on the life history endpoints (growth rate, development time, and head width) for atrazine, carbaryl, and endosulfan. Pesticide concentrations are log transformed. Shown are means \pm SE. Regression lines are given as predicted by the general linear models.

\section{Pesticide stress}

The MANCOVA showed that neither carbaryl (Wilks' $\lambda=0.93, F_{6,123}=1.54, P=0.17$ ) nor its interaction with predation stress (Wilks' $\lambda=0.90, F_{12,246}$ $=1.07, P=0.39$ ) affected the response variables. Atrazine influenced the response of the individuals, but only in interaction with predation risk (Wilks' $\lambda=0.81$, $\left.F_{12,208}=1.86, P=0.040\right)$. Endosulfan had an overall influence on the individuals (Wilks' $\lambda=0.93, F_{6,170}=$ 2.25, $P=0.040$ ), and this influence depended on predation risk (Wilks' $\lambda=0.69, F_{12,340}=5.72, P<$ $0.001)$. As the MANCOVA showed no effect of carbaryl on the response variables, we only report the univariate analyses of each response variable for atrazine and endosulfan. Atrazine did not influence the three life history traits, while endosulfan interacted with predation risk for both growth rate and head width (Table 1). Growth rate of isolated larvae increased with increasing endosulfan concentrations (slope $=0.0069, t_{66}=4.48, P$ $<0.0001$ ), while it decreased in the other two predation risk treatments (pooled slope $=-0.0068, t_{126}=-3.51, P$ $=0.0007$; Fig. 2). Head width decreased with increasing endosulfan concentrations, but only in the larvae reared with a fish predator (slope $=-0.1023, t_{49}=-2.04, P=$
0.04; isolated larvae and larvae reared with conspecifics, pooled slope $=0.084, t_{121}=1.50, P=0.14$; Fig. 2 ).

Overall, food ingestion was not affected by increasing concentrations of atrazine and endosulfan (Table 1, Fig. 3). However, it interacted with predation risk in the endosulfan treatment, showing a significant decrease with increasing endosulfan concentrations in the larvae reared with a fish predator (slope $=-0.3189, t_{69}=-4.08$, $P=0.0001$ ), while food ingestion was not affected by endosulfan in the other predation risk levels (pooled slope $=0.01, t_{127}=0.45, P=0.65$; Fig. 3).

Assimilation efficiency was influenced by both atrazine and endosulfan (Table 1, Fig. 3). It increased with increasing concentrations of atrazine, and this increase was less pronounced in isolated larvae (isolation treatment, slope $=0.0687, t_{45}=2.02, P=0.05$ ) than in larvae from the conspecifics and fish predator treatments (pooled slope $=0.104, t_{86}=2.63, P=0.01$ ). Assimilation efficiency also increased with increasing concentrations of endosulfan, but only in the isolation treatment (slope $=0.0445, t_{65}=2.31, P=0.02$ ), while it decreased in the conspecifics and the fish predator treatments (pooled slope $=-0.132, t_{127}=-3.40, P=0.0009$; Fig. 3 ). 
○ Isolation
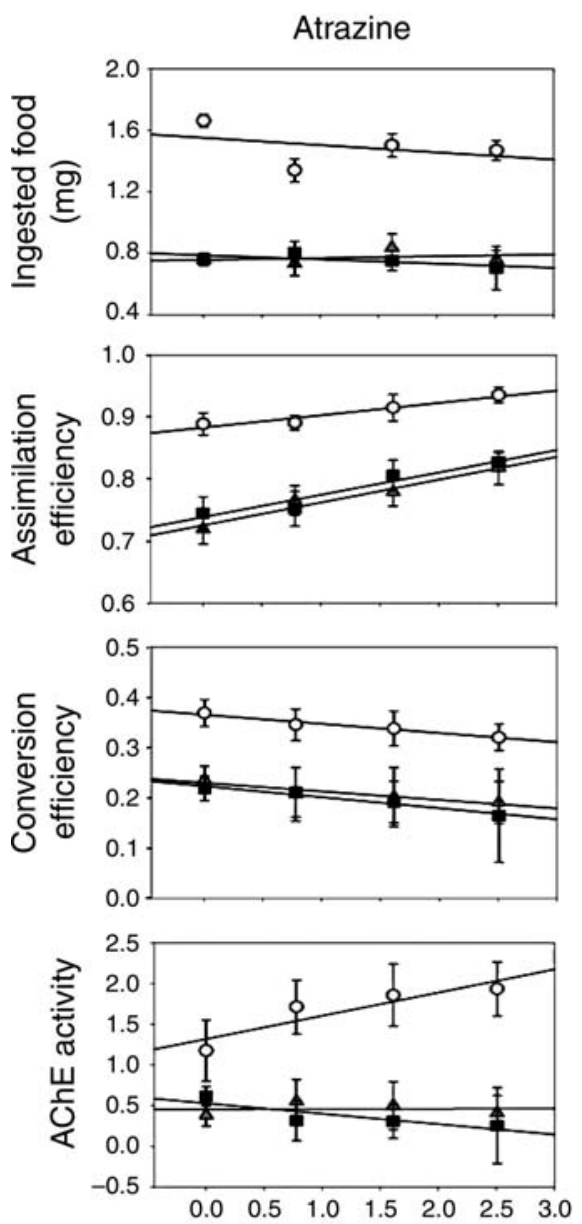

$\Delta$ Conspecifics

Carbaryl
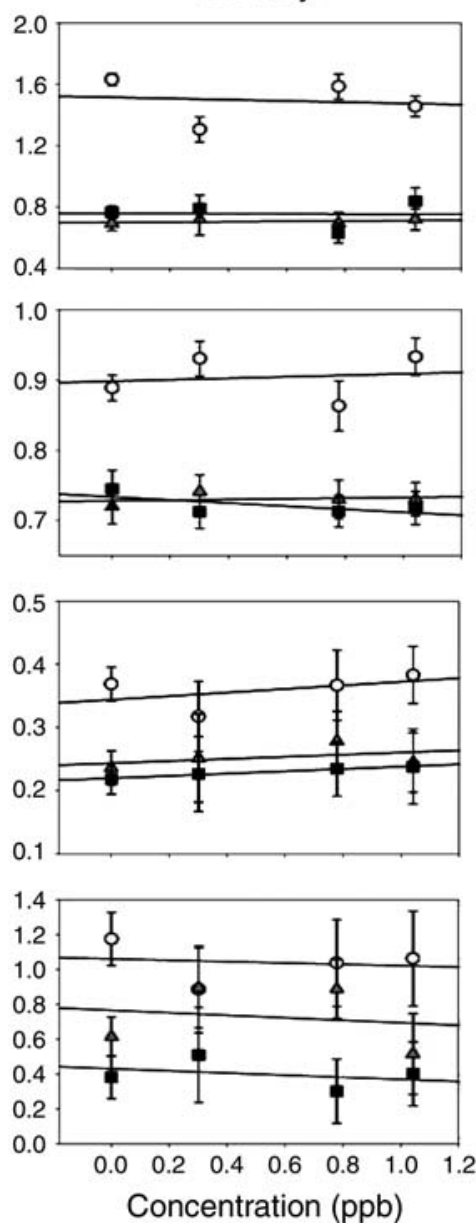

- Conspecifics + fish

Endosulfan
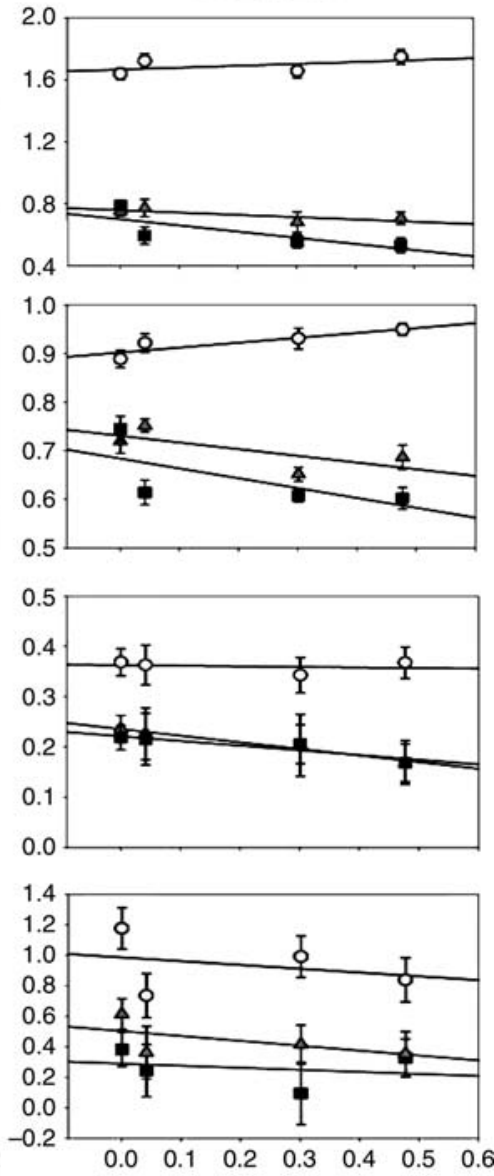

FIG. 3. Effects of pesticide concentrations (plotted in parts per billion, ppb) and predation stress on digestive physiology variables (food ingestion and assimilation and conversion efficiency) and AChE activity ( $\mu$ mol substrate hydrolyzed.min ${ }^{-1}$. $[\mathrm{g} \text { tissue }]^{-1}$ ) for atrazine, carbaryl, and endosulfan. Pesticide concentrations are log-transformed. Shown are means \pm SE. Regression lines are given as predicted by the general linear models.

Conversion efficiency was significantly influenced by atrazine and endosulfan (Table 1, Fig. 3). It decreased with increasing atrazine concentrations, and this decrease did not differ among predation risk levels (pooled slope $\left.=-0.0546, t_{133}=-2.24, P=0.027\right)$. Conversion efficiency decreased with increasing endosulfan concentrations only in the conspecifics and fish predator treatment (pooled slope $=-0.03, t_{125}=-2.04, P=$ 0.049 ), and was not affected by endosulfan in the isolation treatment (slope $=0.004, t_{65}=0.80, P=0.42$; Fig. 3).

Overall, AChE activity did not change with increasing concentrations of the two pesticides, although a significant interaction between atrazine concentrations and predation stress was present (Table 1). Isolated larvae had a higher AChE activity with increasing atrazine concentrations (slope $=0.3941, t_{25}=2.89, P=$ 0.008 ), while AChE activity was not affected by atrazine concentrations in the other predation risk levels (pooled slope $=-0.002, t_{45}=1.24, P=0.22$; Fig. 3).

\section{SEM models}

The SEM model provided good fit to the data for each pesticide (Fig. 4). In general, variation for all endpoints was well explained by the model for all three pesticides ( $R^{2}$ range: $0.52-0.85$ ), with the exception of head width (atrazine, $R^{2}=0.13$; carbaryl, $R^{2}=0.10$; endosulfan, $R^{2}$ $=0.20$ ). For a complete table on direct, indirect, and total effects of pesticide stress, predation risk, and their interaction, see Appendix.

For all three pesticides, the SEM analysis showed a strong, positive direct influence of ingested food onto assimilated food (i.e., as more food is ingested, more food is assimilated; standardized path coefficients range: $0.70-0.90$ ). Assimilated food in turn had a positive direct influence on growth rate (i.e., more assimilated food resulted in higher growth rates; standardized path 

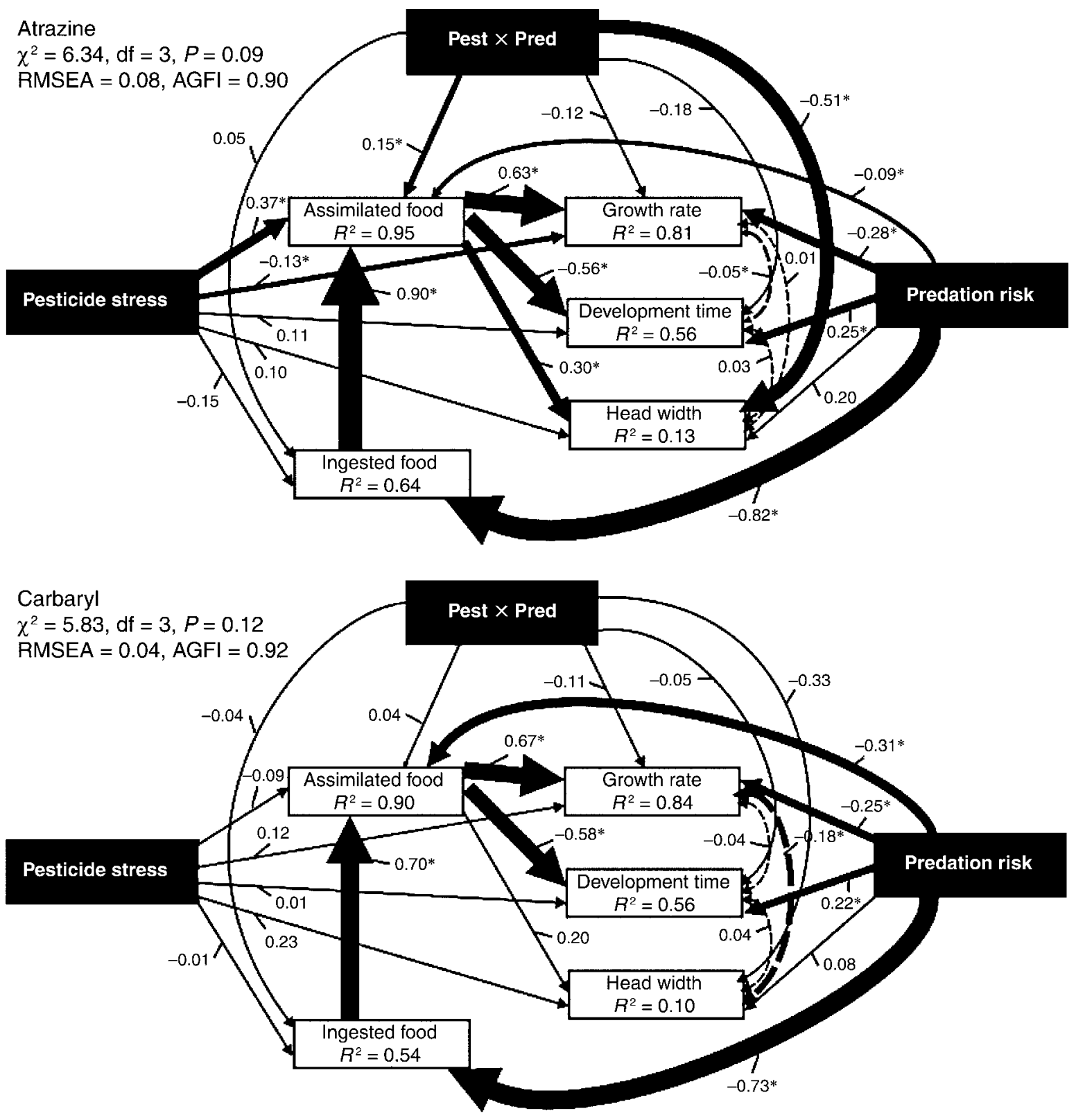

FIG. 4. Graphical representation of the supported SEM model for each pesticide and its goodness-of-fit measures. Standardized path coefficients and variation explained by the model $\left(R^{2}\right)$ are given for response variables. Significant path coefficients $(P<0.05)$ are indicated by asterisks. Thickness of the arrows is proportional to the strength of the standardized path coefficient. Key to abbreviations: Pest $\times$ Pred, pesticide stress $\times$ predation risk; RMSEA, root mean square error of approximation, AGFI, adjusted goodness-of-fit index.

coefficients range: $0.63-0.67)$, a negative direct influence on development time (i.e., more assimilated food resulted in shorter development times; standardized path coefficients range: -0.42 to -0.58 ) and either a positive (atrazine: 0.30 ) or no direct influence (carbaryl and endosulfan) on head width (Fig. 4).

For all three pesticides, predation risk directly decreased food ingestion (standardized path coefficient of the direct link from predation risk to ingested food range: -0.73 to -0.82 , Fig. 4 ). Predation risk also had a negative direct effect on assimilated food (range: -0.09 to -0.31 ), growth rate (range: -0.21 to -0.28 ) and development time (range: $0.22-0.38$, Fig. 4). The direct effect of predation risk on food ingestion cascaded through food assimilation and indirectly induced a further decrease on growth rate (range indirect effects: -0.51 to -0.55 ), head width (range indirect effects: -0.10 to -0.25 ), and increased development time (range indirect effects: $0.43-0.47)$. For all indirect effects, see Appendix. 


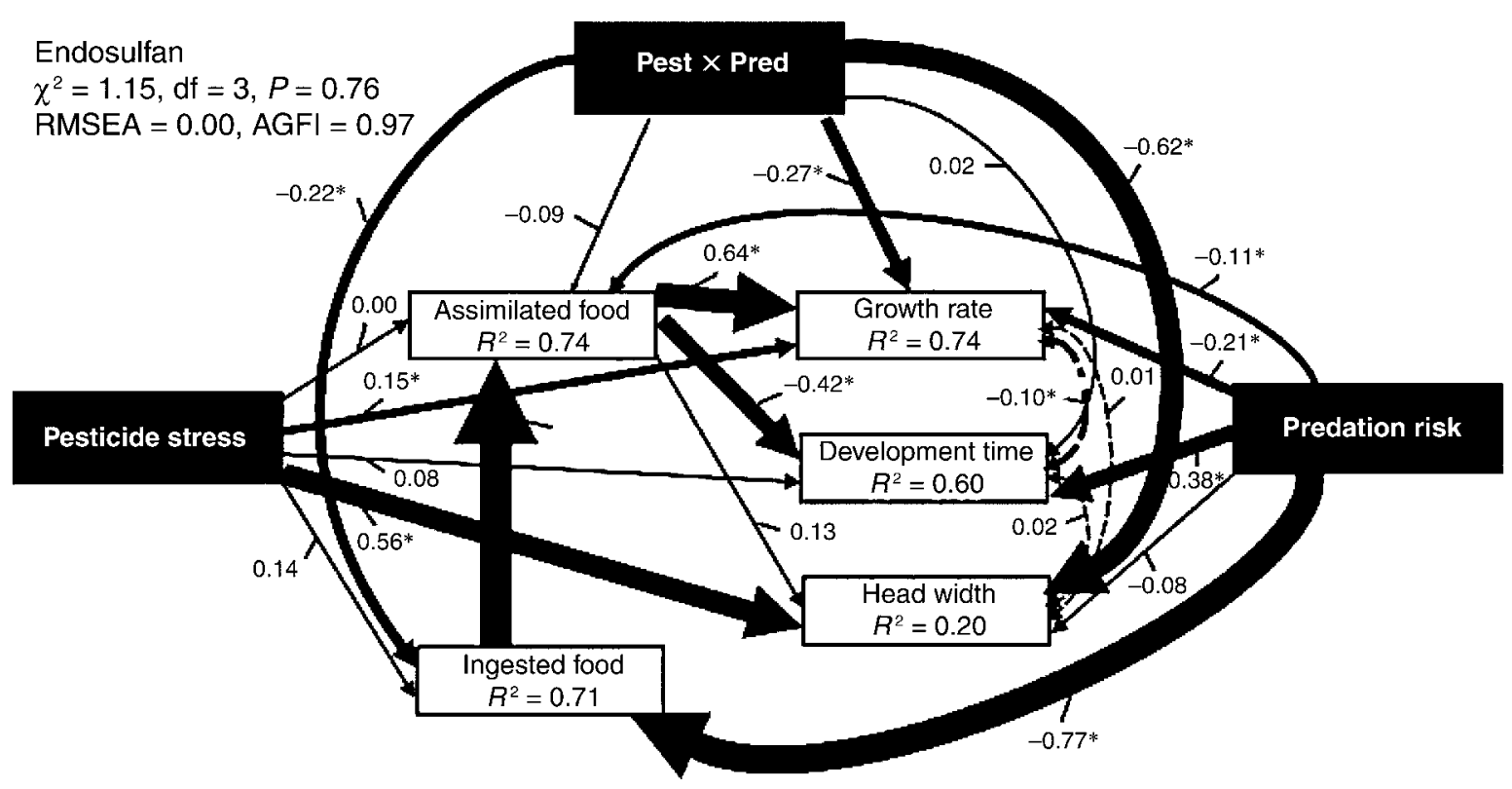

FIG. 4. Continued.

Atrazine directly decreased growth rate $(-0.13$, Fig. 4). However, atrazine also directly increased food assimilation (0.37, Fig. 4), which resulted in an indirect positive effect of atrazine on growth rate $(0.15$, Appendix). The increase in food assimilation under atrazine stress was more pronounced under predation risk, as indicated by the significant pesticide stress $\times$ predation risk interaction (0.15). The negative direct and the positive compensatory indirect effect of atrazine on growth rate cancelled out, resulting in no total effect of atrazine on growth rate (0.02, Appendix). Atrazine decreased head width, but only when combined with predation risk ( -0.51 , Fig. 4). Atrazine also tended to decrease food ingestion and to increase development time although these trends were not significant. When combined with predation risk, atrazine tended to decrease development time, although again this trend was not significant.

Carbaryl had no direct or indirect effect on any of the response variables, neither alone nor in combination with predation risk (Fig. 4, Appendix).

Endosulfan had direct positive effects on growth rate $(+0.15)$ and head width $(+0.56)$. Both effects were counteracted under predation risk (pesticide stress $\times$ predation risk growth rate was -0.27 and head width was -0.62 ). Endosulfan also directly decreased food ingestion, but only under predation risk (pesticide stress $\times$ predation risk, -0.22 , Fig. 4 ).

\section{Discussion}

Overall, negative effects of predation stress were strong while those of the pesticides were small or absent because of compensatory digestive responses. The effects of pesticide stress, predation stress, and their interaction on life history variables worked mainly through indirect effects: food ingestion, food assimilation, and food conversion. Overall, these indirect effects of pesticide exposure and predation risk on life history variables were stronger than the direct effects. Note that the socalled direct effects are effects not explained by food ingestion and food assimilation. Therefore, any process not related with digestion may be the cause of these direct effects. We demonstrated that predation risk in an invertebrate may reinforce pesticide stress by off-setting compensatory metabolic responses.

Predation risk had negative effects on all variables (except head width), with isolated larvae showing more optimal values for all variables than the larvae of the conspecifics and the fish predator treatments. When compared with the isolation treatment, growth rate was reduced 35\% and development time was extended 30\% in the conspecifics and fish treatments. These negative effects on life history could be explained by significant reductions in food intake, assimilation efficiency, and conversion efficiency under predation stress. These results are consistent with studies on other damselfly species (McPeek et al. 2001, Stoks and McPeek 2003, Stoks et al. 2005b). The negative effects of the exposure to conspecifics can be explained by the cannibalistic behavior of damselflies, which therefore impose predation stress (De Block and Stoks 2004). As in another study on coenagrionid damselflies (McPeek et al. 2001), in most cases, no further differentiation between the conspecifics and the fish predator treatment was observed. A differentiation between these two levels of predation risk was found only in the endosulfan treatments, where the presence of a fish predator 
produced a further decrease in head width and food ingestion compared to the conspecifics treatment.

Predation risk did negatively affect AChE activity, with levels being up to $70 \%$ lower in the presence of predation stress. To the best of our knowledge, this is the first demonstration of predation risk inhibiting AChE. According to EPA (2000), a 20\% decrease in AChE activity indicates inhibition of this enzyme, a $50 \%$ decrease already has negative effects on the organism, and a 90\% decrease may be lethal (EPA 2000). Therefore, the combined effect of predation risk and an AChE-inhibiting pesticide may further decrease AChE activity below critical levels. This may contribute to the negative, synergistic mortality effects of pesticides and predation stress (Relyea and Mills 2001). The nature of the link between predation stress and cholinesterase inhibition is unclear. Further research is needed to shed light on this issue.

Effects of pesticide stress on life history were subtle for both atrazine and endosulfan, and were absent for carbaryl. The SEM analyses showed a direct negative effect of atrazine on growth rate (Fig. 4), which was fully compensated by an increase in food assimilation produced by the pesticide. Compensation to chemical stress (i.e., water salinity) by an increase in assimilation has been previously shown in two clam species (Zhuang 2005 , 2006). To the best of our knowledge, this is the first time where it is shown that such compensation can occur in response to pesticide stress. This compensation was more pronounced in the presence of predation risk as indicated by the interaction between atrazine and predation stress. Atrazine exposure reduced conversion efficiency, probably because of reallocation of energy to other processes like detoxification (Lika and Kooijman 2003). The SEM analyses also showed a strong negative effect of atrazine stress on final size (i.e., head width) but only under predation risk (negative path coefficient associated with pesticide $\times$ predation stress). As size is a fixed parameter upon emergence in odonates (Corbet 1999) and is positively correlated with fitness in our study species (Harvey and Walsh 1993), this interaction may have detrimental effects on long term fitness. Note that the ANCOVAs were not able to detect this interaction between both stressors while SEM did. This is because SEM analyses explore not only the effects of the predictor variables but also the structural relationships between endpoint variables of the same individual. This feature of SEM makes it a more accurate tool for researchers, especially when addressing cause-effect relationships.

Despite the fact that carbaryl at 20 ppb strongly increased mortality in a pilot experiment, the used doses in the actual experiment (maximum $10 \mathrm{ppb}$ ) had no significant effect on any development response or digestive variable. Similar doses (i.e., $10 \mathrm{ppb}$ ) also did not affect survivorship or emergence success in other damselflies (Hardersen and Wratten 1998, Hardersen 2000). The lack of a strong effect of the carbaryl treatment on response variables may be due to a combination of acclimatization of the individuals to the treatment, too low doses of carbaryl, and/or too fast degradation. As the analysis of the water samples revealed that rates of carbaryl degradation were up to 90-100\% after seven days, it is likely that the carbaryl exposure was more similar to a spiked than to a chronic exposure. This could have given the larvae enough time between each water renewal to recover from any adverse effect of the carbaryl.

Negative effects of endosulfan were present on growth rate and head width but only under predation stress. Endosulfan increased growth rate in isolated larvae, but when combined with predation stress it produced a strong decrease in growth rate. Studies in other taxa exposed to similar doses of endosulfan, found no effect on this trait (Ribeiro et al. 2001, Rohr et al. 2003). As also shown in the atrazine treatment, a strong negative effect of endosulfan on final size was detected but only at the highest predation risk level (i.e., fish treatment). These patterns can be explained by a compensatory increase in assimilation efficiency under endosulfan stress, which is offset under predation stress. Additionally, while food ingestion was not affected by endosulfan stress in isolated larvae and larvae reared with conspecifics, it decreased with increasing endosulfan concentrations under fish exposure. Decreased food consumption and assimilation efficiency after endosulfan exposure has been shown before in other taxa (Ribeiro et al. 2001) but at considerably higher doses than here (i.e., above 100 ppm).

Pesticide stress, if anything, had positive effects on AChE activity. AChE activity was stimulated by atrazine in the isolation treatment, which contradicts previous studies showing that AChE activity was inhibited by atrazine (Forget et al. 2003). The reasons for this finding remain unclear. Neither carbaryl nor endosulfan affected AChE activity. The effect of carbaryl and endosulfan on this enzyme vary across studies, with even opposite results (Jensen et al. 1997, Dutta and Arends 2003, Rickwood and Galloway 2004).

Notice that the results reported by our study are under low, realistic environmental concentrations for the three pesticides. The emerging picture from a series of studies (Relyea and Mills 2001, Relyea 2004, 2005) is that the synergism between pesticide stress and predation cues may only be present at intermediate, relatively low pesticide concentrations. At too low pesticide concentrations, no effect of the pesticide and only predation stress is observed. At too high concentrations, pesticides may become the most important stressor in the system and mask or override the effects of predation stress on the individuals, resulting in mortality solely explained by pesticide exposure. At the concentrations used in our study, we observed synergistic effects only on life history. It is likely that with increasing concentrations we would have seen synergistic mortality effects until reaching a certain pesticide concentration, 
beyond which mortality would have been explained solely by pesticide exposure.

Our study in an invertebrate adds to the growing list of studies in vertebrates (Boone and Semlitsch 2001, Relyea and Mills 2001, Relyea 2003, Teplitsky et al. 2005) showing that in the absence of predation stress, one may underestimate the effects of pesticides. This interaction between pesticide and predation stress, therefore, has attracted much attention by environmentalists and is suspected to underlie global decline of taxa like amphibians (Storfer 2003). We made progress in understanding the physiological mechanistic base of this interaction, as our data suggest that the balance between behavioral (food intake) and physiological mechanisms (i.e., food assimilation and conversion efficiency) may shape the occurrence of the interaction between pesticide stress and predation risk. Unlimited food availability may have made possible the compensation as observed in the atrazine and endosulfan treatments. At low food levels and higher pesticide doses, compensatory mechanisms may no longer be sufficient to buffer negative conditions and strong negative interactions on life history may become apparent. We could also show that $\mathrm{AChE}$ is inhibited by pesticides and by predation risk. As strong inhibition of AChE causes mortality (Fleischli et al. 2004), it is possible that this mechanism can partially explain the documented synergistic mortality effects between pesticides and predation stress (Relyea and Mills 2001, Relyea 2003).

The followed mechanistic approach may give additional insights about the most probable conditions where an interaction between stressors will occur and can, therefore, help to bridge the gap between laboratory and field studies. Although the pesticide concentrations found in nature are currently mostly below the lethal concentrations for damselflies, our results suggest that negative effects can become apparent under realistic field conditions, especially under high levels of predation stress as found in fish ponds and damselfly populations with high densities. The latter is to be expected as damselfly larvae are cannibalistic and impose predator stress.

\section{AcKnowledgments}

We thank Marjan De Block and two anonymous reviewers for providing constructive comments to improve this manuscript. M. Campero is a SABLam Ph.D. scholar of the Interuniversity Belgian Cooperation. S. Slos is a Ph.D. scholar of the Institute for the Promotion of Innovation through Sciences and Technology in Flanders (IWT-Vlaanderen). This work was financially supported by research grants from FWO (G.0269.04), KULeuven Research Fund (OT/04/23), and EUIP project ALARM (GOCE-CT-2003-506675).

\section{Literature Cited}

Adams, S. M. 2001. Biomarker/bioindicator response profiles of organisms can help differentiate between sources of anthropogenic stressors in aquatic ecosystems. Biomarkers 6:33-44.

Banks, M. J., and D. J. Thompson. 1987. Regulation of damselfly populations: the effects of larval density on larval survival, development rate and size in the field. Freshwater Biology 17:357-365.

Barry, M. J. 2002. Progress toward understanding the neurophysiological basis of predator-induced morphology in Daphnia pulex. Physiological and Biochemical Zoology 75: 179-186.

Boone, M., and R. Semlitsch. 2001. Interactions of an insecticide with larval density and predation in experimental amphibian communities. Conservation Biology 15:228-238.

Boonstra, R., D. Hik, G. R. Singleton, and A. Tinnikov. 1998. The impact of predator-induced stress on the snowshoe hare cycle. Ecological Monographs 68:371-394.

Chang, K. H., M. Sakamoto, and T. Hanazato. 2005. Impact of pesticide application on zooplankton communities with different densities of invertebrate predators: an experimental analysis using small-scale mesocosms. Aquatic Toxicology 72:373-382.

Congdon, J., A. Dunham, W. Hopkins, C. Rowe, and T. Hinton. 2001. Resource allocation-based life histories: a conceptual basis for studies of ecological toxicology. Environmental Toxicology and Chemistry 20:1698-1703.

Corbet, S. 1999. Dragonflies: behaviour and ecology of Odonata. Harley Books, Essex, UK.

De Block, M., and R. Stoks. 2004. Cannibalism-mediated life history plasticity to combined time and food stress. Oikos 106:587-597.

Dewey, S. L. 1986. Effects of the herbicide atrazine on aquatic insect community structure and emergence. Ecology 67:148162.

Dutta, H., and D. Arends. 2003. Effects of endosulfan on brain acetylcholinesterase activity in juvenile bluegill sunfish. Environmental Research 91:157-162.

EPA. 2000. The use of data on cholinesterase inhibition for risk assessment for organophosphorous and carbamate pesticides. Environmental Protection Agency, Washington, D.C., USA.

Fleischli, M. A., J. C. Franson, N. J. Thomas, D. L. Finley, and W. Riley. 2004. Avian mortality events in the United States caused by anticholinesterase pesticides: a retrospective summary of National Wildlife Health Center records from 1980 to 2000. Archives of Environmental Contamination and Toxicology 46:542-550.

Forget, J., B. Beliaeff, and G. Bocquené. 2003. Acetylcholinesterase activity in copepods (Tigriopus brevicornis) from the Vilaine River estuary, France, as a biomarker of neurotoxic contaminants. Aquatic Toxicology 62:195-204.

Gribbin, S. D., and D. J. Thompson. 1990. Asymmetric intraspecific competition among larvae of the damselfly Ischnura elegans (Zygoptera, Coenagrionidae). Ecological Entomology 15:37-42.

Hardersen, S. 2000. Effects of carbaryl exposure on the last larval instar of Xanthocnemis zealandica: fluctuating asymmetry and adult emergence. Entomologia Experimentalis et Applicata 96:221-230.

Hardersen, S., and S. D. Wratten. 1998. The effects of carbaryl exposure of the penultimate larval instars of Xathocnemis zealandica on emergence and fluctuating asymmetry. Ecotoxicology 7:297-304.

Harvey, I. F., and K. J. Walsh. 1993. Fluctuating asymmetry and lifetime mating success are correlated in males of the damselfly Coenagrion puella (Odonata, Coenagrionidae). Ecological Entomology 18:198-202.

Hose, G. C., R. P. Lim, R. V. Hyne, and F. Pablo. 2003. Shortterm exposure to aqueous endosulfan affects macroinvertebrate assemblages. Ecotoxicology and Environmental Safety 56:282-294.

Jensen, C., L. Garsdal, and E. Baatrup. 1997. Acetylcholinesterase inhibition and altered locomotor behavior in the carabid beetle Pterostichus cupreous. A linkage between biomarkers at two levels of biological complexity. Environmental Toxicology and Chemistry 16:1727-1732. 
Jöreskog, K., and D. Sörbom. 2004. LISREL 8.72. Scientific Software International, Lincolnwood, Illinois, USA.

Lika, K., and S. A. L. M. Kooijman. 2003. Life history implications of allocation to growth versus reproduction in dynamic energy budgets. Bulletin of Mathematical Biology 65:809-834.

Lima, S. L. 1998. Stress and decision making under the risk of predation: recent developments from behavioral, reproductive, and ecological perspectives. Advances in the Study of Behavior 27:215-290.

McPeek, M. A. 1990. Behavioral differences between Enallag$m a$ species (Odonata) influencing differential vulnerability to predators. Ecology 71:1714-1726.

McPeek, M. A. 2004. The growth/predation risk trade-off: So what is the mechanism? American Naturalist 163:E88-E111.

McPeek, M. A., M. Grace, and J. M. L. Richardson. 2001. Physiological and behavioral responses to predators shape the growth/predation risk trade-off in damselflies. Ecology 82:1535-1545.

Mills, N. E., and R. D. Semlitsch. 2004. Competition and predation mediate the indirect effects of an insecticide on southern leopard frogs. Ecological Applications 14:10411054.

Newman, M. 1998. Fundamentals of ecotoxicology. Ann Arbor Press, Chelsea, Michigan, USA.

Pauwels, K., R. Stoks, and L. De Meester. 2005. Coping with predator stress: interclonal differences in induction of heatshock proteins in the water flea Daphnia magna. Journal of Evolutionary Biology 18:867-872.

Pugesek, B. 2003. Modeling a complex conceptual theory of population change in the Shiras moose: history and recasting as a structural equation model. Pages 60-84 in B. Pugesek, A. Tomer, and A. Von Eye, editors. Structural equation modeling: applications in ecological and evolutionary biology. Cambridge University Press, Cambridge, UK.

Rao, J. V., K. Parvathi, P. Kavitha, N. M. Jakka, and R. Pallela. 2005. Effect of chlorpyrifos and monocrotophos on locomotor behaviour and acetylcholinesterase activity of subterranean termites, Odontotermes obesus. Pest Management Science 61:417-421.

Relyea, R. 2003. Predator cues and pesticides: a double dose of danger for amphibians. Ecological Applications 13:15151521.

Relyea, R. 2004. Synergistic impacts of malathion and predatory stress on six species of North American tadpoles. Environmental Toxicology and Chemistry 23:1080-1084.

Relyea, R. 2005. The lethal impacts of roundup and predatory stress on six species of North American tadpoles. Archives of Environmental Contamination and Toxicology 48:351-357.

Relyea, R., and N. Mills. 2001. Predator-induced stress makes the pesticide carbaryl more deadly to gray treefrog tadpoles (Hyla versicolor). Proceedings of the National Academy of Sciences (USA) 98:2491-2496.

Ribeiro, S., J. Sousa, A. Nogueira, and A. Soares. 2001. Effect of endosulfan and parathion on energy reserves and physiological parameters of the terrestrial isopod Porcellio dilatatus. Ecotoxicology and Environmental Safety 49:131138.

Rickwood, C., and T. Galloway. 2004. Acetylcholinesterase inhibition as a biomarker of adverse effect. A study of
Mytilus edulis exposed to the priority pollutant chlorfenvinpos. Aquatic Toxicology 67:45-56.

Rohr, J., and P. Crumrine. 2005. Effects of an herbicide and an insecticide on pond community structure and processes. Ecological Applications 15:1135-1147.

Rohr, J., A. Elskus, B. Sheperd, P. Crowley, T. McCarthy, J. Niedzwiecki, T. Sager, A. Sih, and B. Palmer. 2003. Lethal and sublethal effects of atrazine, carbaryl, endosulfan, and octyphenol on streamside salamander (Ambystoma barbouri). Environmental Toxicology and Chemistry 22:2385-2392.

Sandahl, J. F., D. H. Baldwin, J. J. Jenkins, and N. L. Scholz. 2005. Comparative thresholds for acetylcholinesterase inhibition and behavioral impairment in coho salmon exposed to chlorpyrifos. Environmental Toxicology and Chemistry 24: 136-145.

Sibly, R. M., and P. Calow. 1989. A life-cycle theory of responses to stress. Biological Journal of the Linnean Society 37:101-116.

Sih, A., A. Bell, and J. Kerby. 2004. Two stressors are far deadlier than one. Trends in Ecology and Evolution 19:274276.

Steurbaut, W., B. De Smet, I. Theuns, W. De Cooman, G. Goemans, and C. Belpaire. 2003. MIRA T-Report on the environment and nature in Flanders. Flemish Environment Agency (VMM), Brussels, Belgium.

Stoks, R., M. De Block, and M. A. McPeek. 2005a. Alternative growth and energy storage responses to mortality threats in damselflies. Ecology Letters 8:1307-1316.

Stoks, R., M. De Block, F. Van de Meutter, and F. Johansson. $2005 b$. Predation cost of rapid growth: behavioural coupling and physiological decoupling. Journal of Animal Ecology 74: 708-715.

Stoks, R., and M. A. McPeek. 2003. Antipredator behavior and physiology determine Lestes species turnover along the pondpermanence gradient. Ecology 84:3327-3338.

Storfer, A. 2003. Global amphibian declines: future directions. Diversity and Distribution 9:151-153.

Teplitsky, C., H. Piha, A. Laurila, and J. Merila. 2005. Common pesticide increases costs of antipredator defenses in Rana temporaria tadpoles. Environmental Science and Technology 39:6079-6085.

Thompson, H. 1999. Esterases as markers of exposures to organophosphates and carbamates. Ecotoxicology 8:369384.

Weber, C. 1993. Methods for measuring the acute toxicity of effluents and receiving waters to freshwater and marine organisms. EPA/600/4-90/027F, Environmental Protection Agency, Washington, D.C., USA.

Yang, J. 1998. Modeling interaction and non-linear effects: a step-by-step LISREL example. Pages 17-42 in R. E. Schumacker and G. A. Marcoulides, editors. Interaction and non-linear effects in structural equation modeling. Lawrence Erlbaum Associates, Mahwah, New Jersey, USA.

Zhuang, S. 2005. Influence of salinity, diurnal rhythm and daylength on feeding in Laternula marilina Reeve. Aquaculture Research 36:130-136.

Zhuang, S. 2006. The influence of salinity, diurnal rhythm and daylength on feeding behavior in Meretrix meretrix Linnaeus. Aquaculture 252:584-590.

\section{APPENDIX}

Direct, indirect, and total effects of pesticide stress, predation risk, and their interaction on response variables (Ecological Archives A017-084-A1). 